\title{
Host habitat assessment by a parasitoid using fungal volatiles Sven Steiner ${ }^{1}$, Daniel Erdmann ${ }^{1}$, Johannes LM Steidle ${ }^{2}$ and Joachim Ruther*1
}

\author{
Address: ${ }^{1}$ Institut für Biologie, Freie Universität Berlin, Haderslebener Str. 9, 12163 Berlin, Germany and ${ }^{2}$ Tierökologie 220c, Universität \\ Hohenheim, 70593 Stuttgart, Germany
}

Email: Sven Steiner - svenst@zedat.fu-berlin.de; Daniel Erdmann - danielerdmann@gmx.de; Johannes LM Steidle - steidle@uni-hohenheim.de; Joachim Ruther* - ruther@zedat.fu-berlin.de

* Corresponding author

\section{Published: 6 February 2007}

Frontiers in Zoology 2007, 4:3 doi:10.1186/1742-9994-4-3
Received: 10 November 2006

Accepted: 6 February 2007

This article is available from: http://www.frontiersinzoology.com/content/4/I/3

(C) 2007 Steiner et al; licensee BioMed Central Ltd.

This is an Open Access article distributed under the terms of the Creative Commons Attribution License (http://creativecommons.org/licenses/by/2.0), which permits unrestricted use, distribution, and reproduction in any medium, provided the original work is properly cited.

\begin{abstract}
Background: The preference - performance hypothesis predicts that oviposition preference of insects should correlate with host suitability for offspring development. Therefore, insect females have to be able to assess not only the quality of a given host but also the environmental conditions of the respective host habitat. Chemical cues are a major source of information used by insects for this purpose. Primary infestation of stored grain by stored product pests often favors the intense growth of mold. This can lead to distinct sites of extreme environmental conditions (hot-spots) with increased insect mortality. We studied the influence of mold on chemical orientation, host recognition, and fitness of Lariophagus distinguendus, a parasitoid of beetle larvae developing in stored grain.
\end{abstract}

Results: Volatiles of wheat infested by Aspergillus sydowii and A. versicolor repelled female parasitoids in an olfactometer. Foraging $L$. distinguendus females are known to be strongly attracted to the odor of larval host feces from the granary weevil Sitophilus granarius, which may adhere in remarkable amounts to the surface of the grains. Feces from moldy weevil cultures elicited neutral responses but parasitoids clearly avoided moldy feces when non-moldy feces were offered simultaneously. The common fungal volatile I-octen-3-ol was the major component of the odor of larval feces from moldy weevil cultures and repelled female parasitoids at naturally occurring doses. In bioassays investigating host recognition behavior of $L$. distinguendus, females spent less time on grains containing hosts from moldy weevil cultures and showed less drumming and drilling behavior than on non-moldy controls. L. distinguendus had a clearly reduced fitness on hosts from moldy weevil cultures.

Conclusion: We conclude that $L$. distinguendus females use I-octen-3-ol for host habitat assessment to avoid negative fitness consequences due to secondary mold infestation of host patches. The female response to fungal volatiles is innate, suggesting that host-associated fungi played a crucial role in the evolution of host finding strategies of $L$. distinguendus. Research on the role of host-associated microorganisms in the chemically mediated orientation of parasitoids is still at the beginning. We expect an increasing recognition of this issue in the future. 


\section{Background}

The reproductive success of insects is determined not only by the number of eggs females lay but also by the survival and fecundity of their offspring. Therefore, oviposition preference of insects has been predicted to correlate with host suitability for offspring development (preference performance hypothesis [1]). This hypothesis which has been referred to in the literature as the 'mother knows best' principle [2] has originally been developed for herbivorous insects but is assumed to play an important role also in parasitic Hymenoptera [3]. In parasitoids, host organisms are the only source of nutrients for immature stages [4], and thus, parental fitness particularly depends on accurate assessment of the host sites in terms of their potential to sustain the development of their larvae. Thus, adaptation to reliable cues enabling the evaluation of the host patch quality is a selective advantage for females searching for oviposition sites [5].

Successful parasitization requires commonly a series of successive steps with host habitat location, host location, and host recognition being the major elements [6-8]. Apart from physical cues [9], volatile and non-volatile chemicals have been found to be of considerable importance at almost every level of this foraging process $[6,10]$. Compounds involved may emanate from the host, host by-products, host food plants, or organisms closely associated with the host [11-13].

Chemical cues can not only attract but also deter parasitoids from entering host sites or from laying eggs into unsuitable hosts. Foraging decisions of insects are affected by both extrinsic factors like the suitability of resources, presence of natural enemies or competitors, and by intrinsic factors like experience and age of the ovipositing female [10,14-17]. In contrast, little attention has been paid to the investigation of chemicals that reliably indicate unfavorable environmental conditions (e.g., temperature, humidity, $\mathrm{CO}_{2}$ concentration) within the host habitat [3].

The solitary ectoparasitoid Lariophagus distinguendus Förster (Hymenoptera: Pteromalidae) parasitizes immature stages of several stored-product infesting beetle species that develop inside grains and seeds [18,19]. The behavior and chemical cues involved in the host finding process of this species have been extensively examined [20-27]. Volatiles emitted by the larval feces of the granary weevil Sitophilus granarius L. and other hosts mediate longrange orientation of the parasitoid during host habitat location $[20,25]$. Once within the host habitat, females search for reliable cues indicating the presence of their hosts. Again, compounds originating from the larval feces have been shown be crucial for host recognition [24]. On grains infested with the host, $L$. distinguendus females per- form a stereotypic sequence of behavioral elements including series of intense antennal drumming, tapping with the abdominal tip on the surface of the infested grain, and finally drilling with the ovipositor into the grain [22]. After immobilizing the host by injecting paralyzing substances, females of $L$. distinguendus typically lay one single egg onto the surface of the host and the hatching larva develops inside the grain while feeding upon the host [28].

Since L. distinguendus attacks beetles that often lay their eggs in clumps within stored grain, intense infestation of the grain by a larger number of beetles can lead to a zoned increase of temperature and humidity within the microhabitat [29]. These abiotic conditions promote the invasion of astigmatid mites and particularly the growth of mold [30,31]. At a critical level of secondary infestation, however, environmental parameters in these hot-spots deteriorate and cause an increased insect mortality within the patch. Thus, reproductive success of parasitoids attacking host larvae in areas of high secondary infestation should be lower than in areas of light or no secondary infestation. The ability to detect and avoid these suboptimal oviposition areas would therefore improve the fitness of L. distinguendus.

Fungi are well-known to release a multitude of volatile organic compounds originating from different metabolic pathways. The composition of these volatile profiles depends on the fungal species as well as on the growing medium and abiotic factors [32]. However, a variety of chemicals is shared by several species and is reliably released under various conditions. Among these widespread fungal volatiles is a group of aliphatic eight-carbon compounds including 1-octen-3-ol, 3-octanone, and 3octanol [33] that are derived from enzymatic degradation of unsaturated fatty acids [34]. In most species 1-octen-3ol occurs as major component, and it has been suggested to use this chemical for the detection of mold infestation in stored grain as well as in living and working environments [35-38].

In the present study, we investigated whether mold infestation of $S$. granarius cultures affects chemical orientation, host recognition behavior, and fitness of L. distinguendus. Mold species studied were Aspergillus sydowii (Bainier and Sartory) Thom and Church and A. versicolor (Vuillemin) Tiraboschi. Furthermore, we determined the amounts of typical eight-carbon fungal volatiles in the weevil cultures and tested the response of female parasitoids to synthetic 1-octen-3-ol. The possible function of fungal volatiles as indicators of suboptimal host patches is discussed. 


\section{Results \\ Influence of mold infestation on host finding behavior}

$L$. distinguendus females avoided the odor of moldy wheat in an four-chamber olfactometer irrespective of the Aspergillus species used to inoculate the grain. In both Aspergillus species the odor of uninfested wheat was significantly preferred over inoculated grain (Figure 1a). Parasitoids were strongly arrested by the odor of larval feces from weevil cultures without mold (Figure 1b). However, larval feces from moldy weevil cultures were no longer preferred by the parasitoids when tested against filter paper as a control. The odor of feces from both the $A$. sydowii and $A$. versicolor cultures was neither significantly preferred nor avoided (A. sydowii: $P=0.76, A$. versicolor: $P$ $=0.058$ ) (Figure 1c). However, when feces from moldy and non-moldy cultures were offered simultaneously, females clearly avoided the feces from the moldy cultures in both cases (Figure 1d).

Influence of mold infestation on host recognition behavior Arrestment time of female parasitoids was significantly decreased on wheat grains from A. sydowii- and A. versicolor-infested weevil cultures when compared to the nonmoldy control grains (Figure 2a). Moreover, characteristic elements of the host recognition behavior (drumming and drilling) were shown less often on grains from the moldy weevil cultures than on control grains (Figure $2 \mathrm{~b}-$ c). No significant differences were found between grains from weevil cultures infested by $A$. sydowii and A. versicolor, respectively (arrestment time: $U=145.5, P=0.14$; drilling: $U=153, P=0.20$; drumming: $U=138, P=0.09)$.

\section{Influence of mold infestation on parasitoid fitness}

Females of L. distinguendus parasitized hosts from both moldy and non-moldy weevil-cultures. However, the number of offspring was significantly lower when host larvae originated from moldy weevil cultures infested with either A. sydowii or A. versicolor as compared to nonmoldy cultures (Figure 3 ). There was no significant difference in the number of offspring between the two mold species $(P=0.43)$. Body size as measured by the length of the tibiae was significantly affected by the infestation with mold. Parasitoids that had developed on hosts from nonmoldy weevil cultures had longer tibiae (female: 0.579 $\mathrm{mm} \pm 0.003$; male: $0.5058 \mathrm{~mm} \pm 0.005)$ than individuals from A. sydowii- (female: $0.571 \mathrm{~mm} \pm 0.003$; male: 0.5058 $\mathrm{mm} \pm 0.003$ ) or $A$. versicolor-moldy cultures (female: $0.573 \mathrm{~mm} \pm 0.003$; male: $0.500 \mathrm{~mm} \pm 0.003)$. Moreover, females of $L$. distinguendus had generally longer tibiae than males. There was no interaction observed between treatment and sex (Table 1).
Volatile analysis of feces from moldy and non-moldy weevil cultures

Fecal volatiles from moldy and non moldy weevil cultures were collected by closed loop stripping and analyzed by coupled gas chromatography-mass spectrometry (GC$\mathrm{MS})$. The major eight-carbon fungal volatile detected in larval feces from moldy weevil cultures was 1-octen-3-ol accompanied by lower amounts of 3-octanone, and 3octanol (Table 2). In larval feces from non-moldy host cultures all compounds were found only in traces. The headspace of $A$. sydowii contained additionally some sesquiterpenes as minor compounds.

\section{Response to synthetic fungal volatile}

L. distinguendus females avoided significantly synthetic 1octen-3-ol at doses between 1500 to 300 ng (Figure 4). At a dose of $30 \mathrm{ng}$, however, parasitoids were neither repelled nor attracted to the synthetic chemical $(P=0.62)$.

\section{Discussion}

To study the ability of parasitoids to use chemical cues for the assessment of host habitat quality, the response of $L$. distinguendus was tested to grains and host feces infested with the two mold species A. sydowii or A. versicolor. Both species co-occur with the parasitoid's hosts in its natural stored grain habitat under humid and warm conditions. The olfactometer experiments clearly demonstrated that naïve females of $L$. distinguendus avoid the odor of grains infested by either mold species when offered simultaneously with healthy grains. Likewise, odor from moldy host feces was avoided when non-moldy host feces were offered as an alternative. Obviously, A. sydowii and A. versicolor release volatiles that are repellent for females of $L$. distinguendus. As shown in experiments in which nonmoldy and moldy feces were tested against filter paper only, the repellent effect of volatiles released by both mold species was even strong enough to neutralize the attractive effect of the odor released by non-moldy host feces. In agreement with these results, host recognition behavior of wasps was much more intense on weevilinfested grains from non-moldy cultures when compared to those from moldy ones. Thus, in a bulk of weevilinfested stored grain containing patches with and without mold, parasitoids would most likely orientate towards non-moldy areas during the host finding process and prefer hosts in non-moldy grains for oviposition.

Chemical analyses and further olfactometer tests pointed to 1-octen-3-ol as a chemical cue that might be responsible for the avoidance response of the parasitoid. This compound was emitted from moldy feces in amounts that repelled $L$. distinguendus females when applied as synthetic chemical to filter paper. Lower concentrations of 1octen-3-ol, comparable to the amounts released by feces from cultures with no visible mold, had no effect on the 


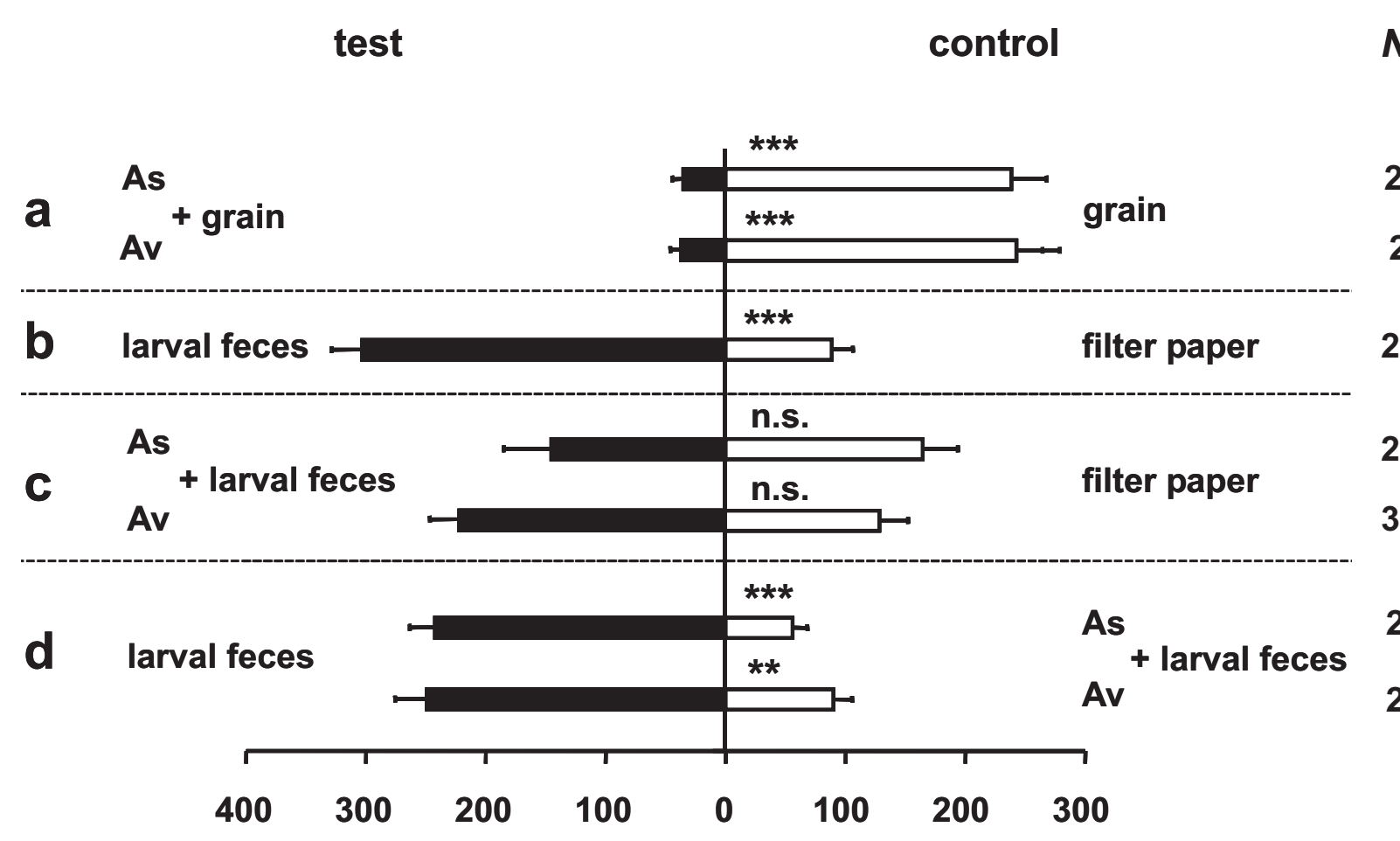

\section{arrestment time [s]}

Figure I

Response of $\boldsymbol{L}$. distinguendus to moldy grain and host feces. Mean arrestment time $( \pm \mathrm{SE})$ of $L$. distinguendus females in the odor fields above test and control chamber of a two choice olfactometer during a 10 min observation period. N.s. $=$ not significant; asterisks indicate significant differences at $P<0.01$ (**) or $P<0.001$ (***) (generalized linear model).

wasps. Therefore, results of experiments using feces and synthetic 1-octen-3-ol match very well provided that release rates were comparable. However, also the other eight-carbon volatiles and further compounds may have contributed to the avoidance response of the parasitoids. Interestingly, 1-octen-3-ol is released as a major volatile not only by the two Aspergillus species studied here but also by numerous fungi from other taxa $[32,33,37,39,40]$. Thus, 1-octen-3-ol reliably indicates fungal growth in stored grain.

The ultimate reason for the avoidance of fungal odor by the parasitoid is probably a reduced fitness, as shown here by a lower number of offspring on hosts from moldy weevil cultures. This might be due to one or several of the following mechanisms: (1) Female parasitoids laid a reduced number of eggs on hosts from moldy cultures. This hypothesis is supported by the fact that important elements of the host recognition behavior (arrestment, drumming, and drilling) were shown to a significantly lesser extend when host-infested grains from moldy weevil cultures were offered. Thus, moldy grains might be less attractive for oviposition by L. distinguendus. (2) Parasi- toids had a higher pre-emergence mortality due to mycotoxins. Numerous mold species including the genus Aspergillus are known to synthesize toxic metabolites $[41,42]$. These might have decreased the parasitoid's and/ or the host's survival rate. (3) Competition between mold and host larvae for wheat nutrients decreased host performance allowing a lower number of parasitoid larvae to develop into adults. It is known from many studies that parasitoid larvae developing on suboptimal hosts may be subjected to an increased pre-emergence mortality as well as a reduced longevity or fecundity [[3] and references therein]. Parasitoids from moldy weevil cultures were significantly smaller supporting the hypothesis that the hosts were not of equal quality. A correlation between body size and the reproductive success has been reported for $L$. distinguendus [43]. Larger females were shown to produce more offspring than smaller individuals because they lived longer and were more fertile. In contrast, size was less important for males. Thus, mold infestation might also influence the number of offspring in the second generation resulting in a decreased inclusive fitness. However, it has to be tested whether the subtle differences in body size observed in this study can actually influence fitness of 

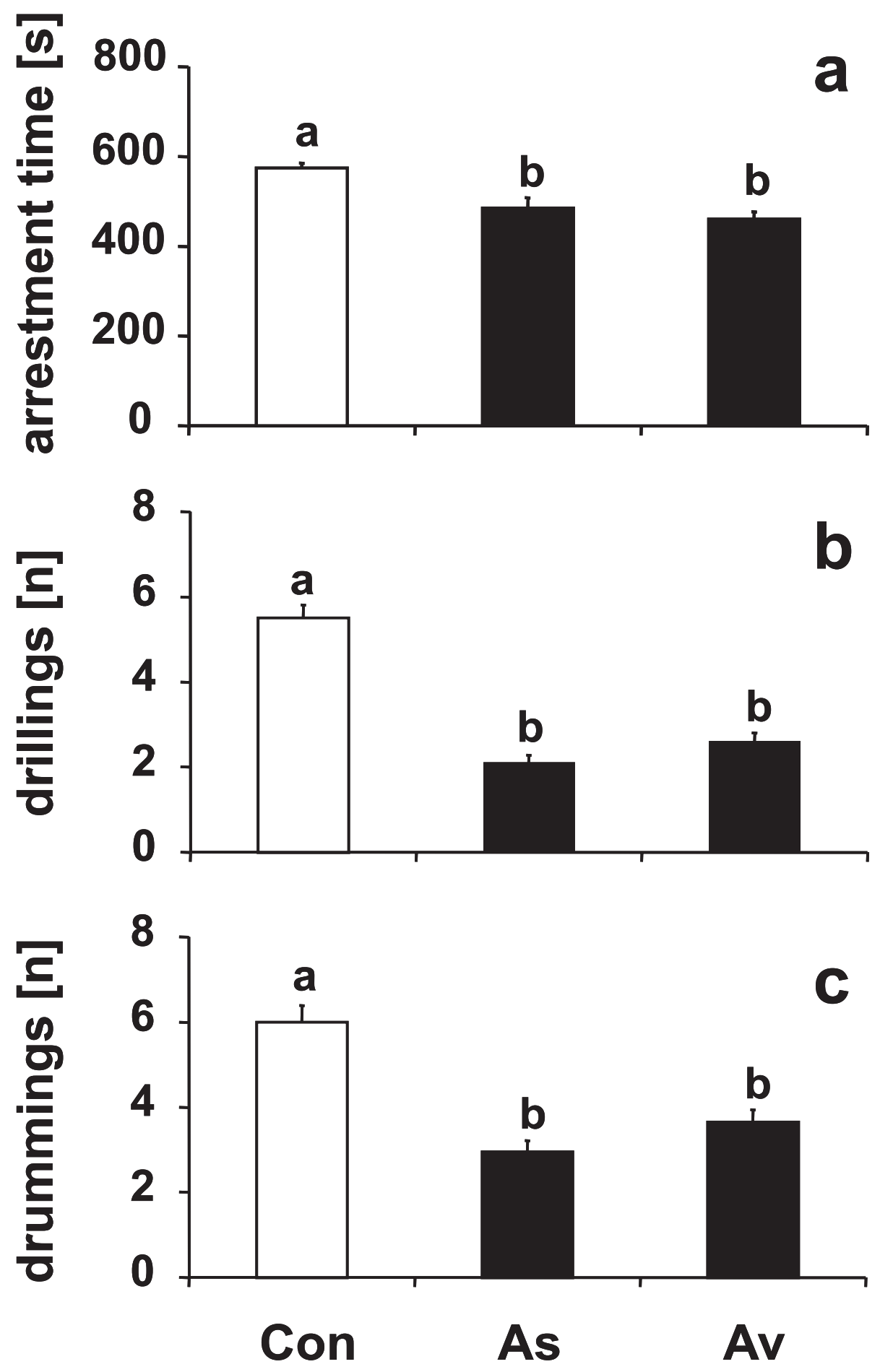

Figure 2

Host recognition behavior of $L$. distinguendus towards hosts from moldy and non-moldy weevil cultures. Response of $L$. distinguendus females to host-infested grains originating from non-moldy weevil cultures (Con) and those infested by $A$. sydowii (As) or A. versicolor (Av). (a) Mean arrestment time on the grain ( \pm SE), (b) number of drumming series ( \pm $\mathrm{SE})$, and (c) number of drillings ( \pm SE) during a 10 min observation period. Bars with different lowercase letters are significantly different at $P<0.00$ I (Kruskal-Wallis $H$-test followed by Bonferroni-corrected Whitney-Mann $U$ tests for multiple comparisons; $N=20$ ). 
Table I: Statistical analysis of tibia lengths of male and female offspring

\begin{tabular}{lcccc}
\hline Source & df & MS & F & P \\
\hline Treatment (Con, As, Av) & 2 & 0.5429 & 722.706 & $<0.001$ \\
Sex (m, f) & 1 & 0.0030 & 3.950 & $\mathbf{0 . 0 2 0}$ \\
Treatment Sex & 2 & 0.0001 & 0.154 & 0.857 \\
Error & 391 & 0.00075 & &
\end{tabular}

Two-way ANOVA of tibia lengths of $L$. distinguendus males $(\mathrm{m})$ and females $(\mathrm{f})$ developing in non-moldy weevil cultures (Con) and weevil cultures infested by A. sydowii (As) or A. versicolor (Av) (df: degrees of freedom; MS: mean squares).

the parasitoids. (4) Secondary infestation by Aspergillus mold caused unfavorable environmental conditions for parasitoid and/or host development. Local mass breeding of insects in stored grain and subsequent mold infestation can lead to distinct areas with extreme abiotic conditions. The development of such hot-spots has been described in detail [29]. Initially, the metabolic activity of the primary pest leads to a moderate increase of temperature and humidity providing optimal conditions for moisture-sensitive secondary pests like astigmatid mites and mold. With increasing growth of fungi, however, moisture and temperature as well as the concentration of carbon dioxide may reach levels that cause a decline of insect populations and finally a breakdown of the hot-spot [29]. $L$. distinguendus and some of its hosts clearly prefer lower humidities over those commonly found in hot spots $[44,45]$. Parasitoid females may recognize at an early stage the development of such unfavorable environmental conditions by avoiding fungal volatiles like 1-octen-3-ol. Thereby, they reduce negative fitness consequences resulting from secondary mold infestation of host patches. Nev-

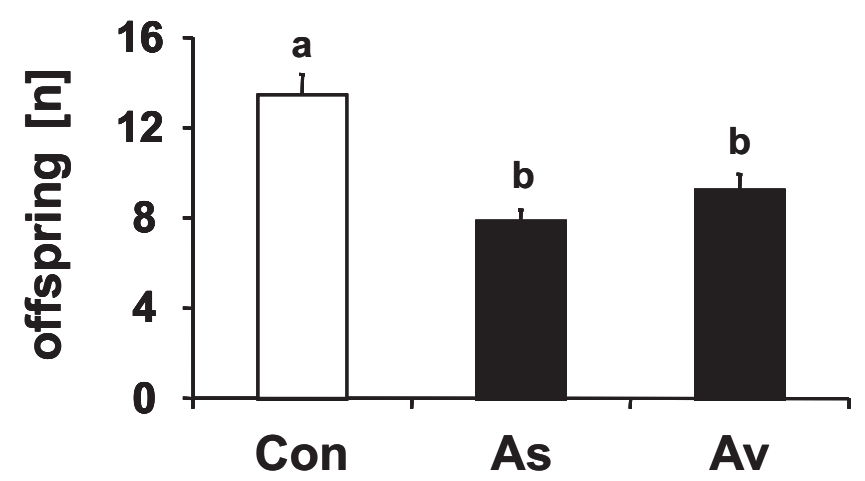

Figure 3

Fecundity of $L$. distinguendus females on hosts from moldy and non-moldy weevil cultures. Mean number of offspring $( \pm S E)$ of $L$. distinguendus females that parasitized host-infested grains from non-moldy weevil cultures (Con) and those infested by $A$. sydowii (As) or A. versicolor (Av). Bars with different lowercase letters are significantly different at $P$ $<0.0$ I (one-way ANOVA; $N=24-26$ ). ertheless, parasitoids are able to develop in hosts from moldy weevil cultures. Therefore, it makes sense that there was a tendency of females to prefer the odor of larval feces from moldy weevil cultures (A. versicolor) in absence of the more attractive alternative, i.e., feces from non-moldy host cultures. The fact that the response of females to the fungal volatile is innate, suggests that host-associated fungi played a crucial role in the evolution of host finding strategies in L. distinguendus.

\section{Conclusion}

The present study is a good example for the 'mother knows best' principle [2] in parasitoids demonstrating that predictions of the preference - performance hypothesis originally made for phytophagous insects may also apply for carnivores. Furthermore, the study is one of the very few demonstrating that infochemicals used by parasitoids for host habitat assessment are produced by hostassociated microorganisms. Hitherto, host associated bacteria $[46,47]$ or fungi $[48]$ have only been shown to produce kairomones that attract parasitoids to their hosts rather than helping them to avoid suboptimal host patches.

Current research on plant-herbivore systems discovers more and more the important role of plant pathogens that influence larval performance and oviposition decisions [2,49-51]. However, only few studies have addressed the question whether plant pathogens may also influence the pattern of herbivore-induced plant volatiles and thereby trigger the response of parasitoids in tritrophic interactions $[52,53]$. Research on the direct or indirect role of microorganisms in the chemically mediated orientation of parasitoids is still at the beginning. We expect an increasing recognition of this issue in the future.

\section{Methods \\ Insect cultures}

Insect cultures were kept at constant conditions of $25^{\circ} \mathrm{C}$, $65 \%$ relative humidity (RH), and a daily light:dark cycle of 16:8 h. The L. distinguendus strain used in the experiments was collected on Stegobium paniceum L. in a flour mill in Uzwill, Switzerland. In our laboratory the parasi- 
Table 2: Amounts of typical eight-carbon fungal volatiles in larval host feces

\begin{tabular}{lccc}
\hline Compound & Con & As & Av \\
\hline l-octen-3-ol & $17 \pm 4$ & $516 \pm 5$ & $730 \pm 46$ \\
3-octanone & $19 \pm 4$ & $116 \pm 14$ & $272 \pm 47$ \\
3-octanol & $5 \pm 1$ & $14 \pm 2$ & $120 \pm 7$ \\
\hline
\end{tabular}

Mean amounts (ng per sampling $\pm \mathrm{SE}, \mathrm{N}=3$ ) of typical eight-carbon fungal volatiles from the headspace of larval feces from non-moldy weevil cultures (Con) and weevil cultures infested by $A$. sydowii (As) or $A$. versicolor (Av).

toid was reared on larvae ( $3^{\text {rd }}$ and $4^{\text {th }}$ instar) of the granary weevil Sitophilus granarius in wheat grains (Triticum aestivum L., var. Batis), as described elsewhere [20].

\section{Insects for experiments}

For all experiments except for experiment 2, naïve L. distinguendus females were used, i.e., individuals without mating and oviposition experience. For this purpose, parasitoids were collected immediately after emergence and held in groups of at most 15 individuals in Petri dishes lined with moistened filter paper. To obtain experienced parasitoids, freshly emerged females were kept together with males in a Petri dish containing host-infested grains for parasitization. After three days, females were removed and kept without hosts in Petri dishes on moistened filter paper. One hour before experiments, L. distinguendus were isolated in $1.5 \mathrm{ml}$ microcentrifuge tubes for acclimation at room temperature. Behaviors shown by the parasitoids during experiments were recorded by using the computer software The Observer 3.0 (Noldus, Wageningen, The Netherlands).

\section{Fungal cultures}

The mold species A. versicolor (Strain no.: BBA 72388) and A. sydowii (Strain no.: BBA 72389) used in the experiments were obtained from the culture collection of the Biolo-

\section{1-octen-3-ol}

control

$\mathbf{N}$

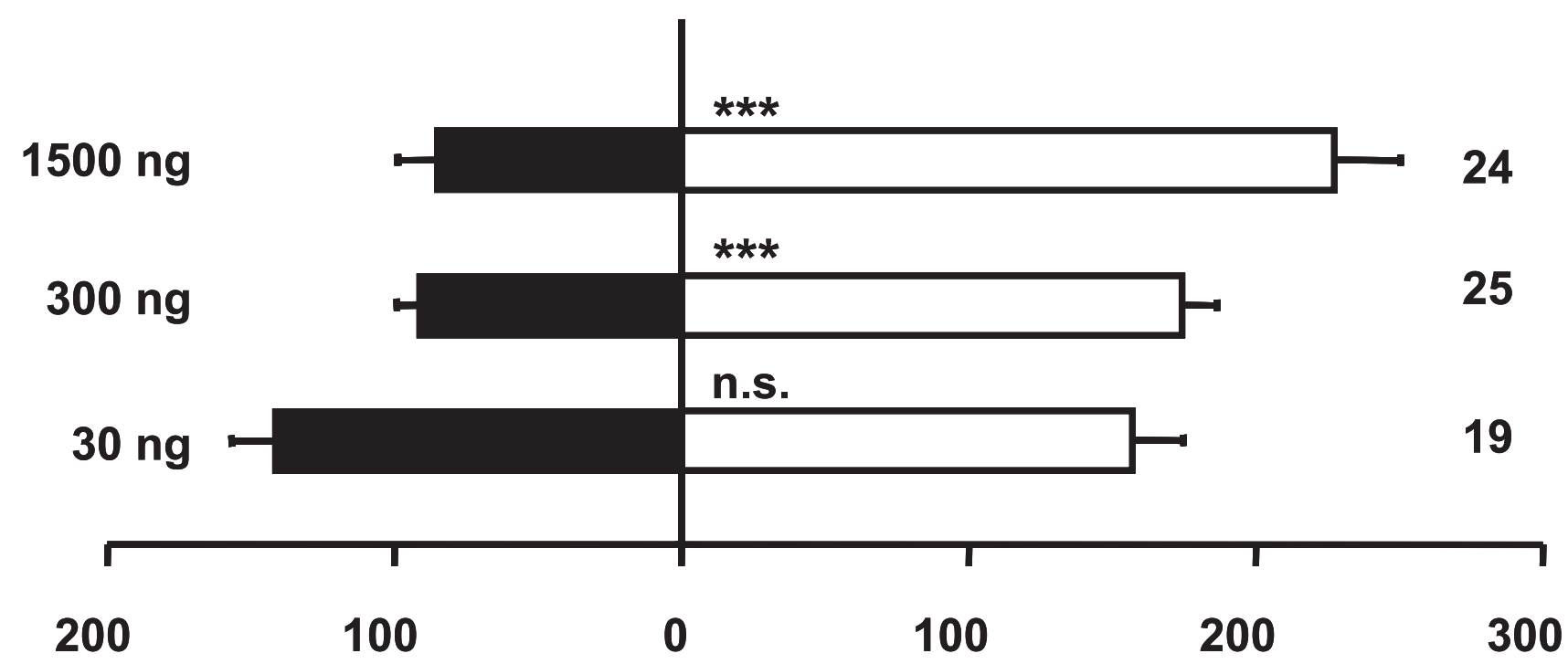

\section{arrestment time [s]}

\section{Figure 4}

Response of $L$. distinguendus to synthetic I-octen-3-ol. Mean arrestment time $( \pm \mathrm{SE})$ of $L$. distinguendus females in the odor fields above test and control chamber of a two choice olfactometer during a 10 min observation period. Test fields were treated with synthetic I-octen-3-ol at different doses; control fields were treated with pure solvent. N.s. = not significant; $* * *$ indicates significant differences at $P<0.001$ (generalized linear model). 
gische Bundesanstalt für Land- und Forstwirtschaft, Institute of Plant Virology, Microbiology, and Biosafety, Berlin, Germany. The isolates originated from intense weevil- and fungi-infested grain cultures of our institute.

Stock cultures of A. sydowii and A. versicolor were freezedried and stored at $4{ }^{\circ} \mathrm{C}$. Subcultures were cultivated on nutrient deficient agar SNA at room temperature for 1 to 2 weeks. A $10 \mathrm{ml}$ portion of sterile water was added to the subculture and the surface was scraped off until conidia were suspended. The suspension was filtered through a folded filter paper (S\&S 595, Schleicher \& Schuell, Dassel, Germany) and the conidia concentrations of the Aspergillus species were determined by counting in a Bürker counting chamber and adjusted with sterilized water to $1.5 \times 10^{6}$ conidia per ml.

\section{Preparation of moldy grain and weevil cultures}

Three batches of wheat grain ( 750 g each) were autoclaved for $30 \mathrm{~min}$. Two batches were treated with $45 \mathrm{ml}$ conidia suspension of A. sydowii and A. versicolor, respectively. For control, the third batch of grain was treated with $45 \mathrm{ml}$ sterilized water. The three batches of grain were incubated separately for four weeks in desiccators at $25^{\circ} \mathrm{C}$. The humidity within the desiccators was adjusted to $65 \%$ by a saturated solution of ammonium nitrate [54]. After this incubation period, mold was visible in the two treated batches of wheat but not in the control. Adult $S$. granarius $(75 \mathrm{ml})$ were added to each batch of grain, allowed to lay eggs into the grains for seven days and subsequently removed. Larval feces used for the olfactometer bioassays and the chemical analysis were obtained by sieving the grain of treated and control cultures 21 to 28 days after the adults had been removed. After removal of the larval feces, weevil-infested grains of mold-treated and control cultures were used to investigate the influence of mold on the host recognition behavior of $L$. distinguendus in experiment 2 and fitness of the parasitoids in experiment 3.

\section{Four-chamber olfactometer}

The response of $L$. distinguendus females to different odor samples was examined using a static four-chamber olfactometer [20,21]. The olfactometer consisted of a cylinder made of acrylic ( $4 \mathrm{~cm}$ high, $\varnothing 19 \mathrm{~cm}$ ) divided into four chambers by crosswise-arranged vertical plates. The top of the cylinder was covered by a plastic gauze (mesh 0.1 $\mathrm{mm}$ ) functioning as a walking arena for the parasitoids. A lid consisting of a plastic ring ( $4 \mathrm{~mm}$ high, $19 \mathrm{~cm} \varnothing$ ) and a glass plate was placed on top of the cylinder to prevent the parasitoids from escaping. Odor samples were placed in one of the chambers (test chamber) using a Petri dish $(5.5 \mathrm{~cm} \varnothing)$. The opposite chamber was used as control chamber and the remaining two chambers adjacent to the test chamber were considered as buffer zones. A single female was released into the center of the walking arena and the time the parasitoid spent in the field above the test or control chamber was recorded for 10 minutes. Parasitoids that were motionless more than $50 \%$ of the total observation time were assumed to be unmotivated and excluded from statistical analysis. To avoid biased results due to possible side preferences of the parasitoids, the position of the samples and the controls was rotated clockwise after each test. Walking arena and glass plate were regularly cleaned with ethanol and demineralized water. Odor sources were exchanged after five individuals had been tested.

\section{Experiment I: Influence of mold infestation on host finding behavior}

The following odor samples were offered in the olfactometer: (A) 10 g grain infested with A. sydowii or A. versicolor vs. $10 \mathrm{~g}$ untreated grain ( $N=20$ for each treatment), (B) $200 \mathrm{mg}$ larval feces from the non-moldy control culture vs. a piece of brown filter paper $(N=21)$, (C) $200 \mathrm{mg}$ larval feces from the mold-infested weevil cultures (A. sydowii: $N=21, A$. versicolor: $N=31$ ) vs. brown filter paper, and (D) $200 \mathrm{mg}$ larval feces from the mold-infested weevil cultures (A. sydowii: $N=21$, A. versicolor: $N=21$ ) vs. 200 mg larval feces from the non-moldy control culture.

\section{Experiment 2: Influence of mold infestation on host recognition behavior}

The host-recognition behavior of experienced L. distinguendus females was examined in a bioassay chamber (10 $\mathrm{mm} \varnothing \times 3 \mathrm{~mm}$ high) made from acrylic [55]. Single grains from weevil cultures infested with $A$. sydowii or $A$. versicolor or those from the control culture were presented to individual females. Arrestment time on the grains and characteristic elements of the host-recognition behavior (number of drumming series and drilling) were recorded for $10 \mathrm{~min}$ using a stereomicroscope under illumination of a microscope light. Grains were exchanged after every parasitoid tested ( $N=20$ for each treatment).

\section{Experiment 3: Influence of mold infestation on parasitoid fitness}

This experiment was done to estimate the offspring production of $L$. distinguendus females in a no-choice situation with hosts from moldy and non-moldy weevil cultures. Virgin females were allowed to copulate with a male and subsequently placed individually in Petri dishes containing weevil-infested grains from the moldy weevilcultures (A. sydowii: $N=25$, A. versicolor: $N=23$ ) or the control culture $(N=25)$. Weevil-infestation of grains was equal in moldy (A. sydowii: $82 \%$, A. versicolor: $76 \%$ ) and non-moldy $(78 \%)$ weevil cultures. Parasitoids were allowed to oviposit until their death. Offspring was assessed by counting the emerged parasitoids after 30 days. In addition, hind tibia length $[56,57]$ was used to measure the body size of randomly selected individuals 
from the offspring of the three treatments (A. sydowii: $N=$ 102, A. versicolor: $N=91$, control: $N=143$ ).

\section{Experiment 4: Response to synthetic I-octen-3-ol}

The response of $L$. distinguendus females to the typical fungal volatile 1-octen-3-ol (98\%, Sigma-Aldrich, Steinheim, Germany) was investigated in another olfactometer experiment. The chemical was applied at different doses [1500 $\mathrm{ng}(\mathrm{N}=24), 300 \mathrm{ng}(N=25)$, and $30 \mathrm{ng}(N=19)]$ to filter paper discs $(\varnothing 4 \mathrm{~cm}$, Melitta, Minden, Germany) and offered in the test chamber of the olfactometer. Paper discs treated with the pure solvent $(30 \mu \mathrm{l}$ dichloromethane) were used as control.

\section{Volatile collection}

Volatiles emitted from the larval feces of the three different weevil-cultures ( $N=3$ for each treatment) were collected by closed loop stripping (CLS) as described elsewhere [21]. Volatiles from $5 \mathrm{~g}$ larval feces were collected for $4 \mathrm{~h}$ on a $1 \mathrm{~mm}$ charcoal layer $(5 \mathrm{mg})$ of a CLSadsorption tube $(65 \mathrm{~mm}$ length $\times 5 \mathrm{~mm} \varnothing)$ (Gränicher $\&$ Quartero, Daumazan, France). The volatiles were eluted with $25 \mu$ dichloromethane containing $5 \mathrm{ng} / \mu \mathrm{l}$ methyl undecanoate as an internal standard and used for chemical analysis by GC-MS.

\section{GC-MS analysis}

Volatile extracts were analyzed by GC-MS using a Fisons 8060 GC (Fisons Instruments) equipped with a $30 \mathrm{~m} \times$ $0.32 \mathrm{~mm}$ ID $\times 0.25 \mu \mathrm{m}$ film thickness DB-5 ms column (J \& W Scientific, Folsom, CA, USA) and coupled to a Fisons MD800 quadrupole MS operated in the electron impact (EI) mode at $70 \mathrm{eV}$. Helium was used as carrier gas at a head pressure of $10 \mathrm{kPa}$. The oven temperature was $40^{\circ} \mathrm{C}$ for $4 \mathrm{~min}$ and then rose to $280^{\circ} \mathrm{C}$ at a rate of $5^{\circ} \mathrm{C} / \mathrm{min}$. The final temperature was held for $10 \mathrm{~min}$ for thermal cleaning of the column. Chemicals were identified by comparison of mass spectra and retention times with those of synthetic reference compounds (Sigma-Aldrich, Steinheim, Germany). For quantification of selected compounds, the peak area of each volatile was related to the peak area of the internal standard.

\section{Statistical analysis}

The arrestment times of female parasitoids spent in test and control field of the olfactometer (experiments 1 and 4) were analyzed by a general estimator equation-generalized linear model (GEE-GLM, SAS version 9.1, PROC GENMOD). Model fitting was carried out with a Poisson error distribution and log link function. Residuals were inspected for normality. Since odor samples in these experiments were renewed after every five insects, we tested not only the factor treatment but also the factors sample and order as explanatory variables for the dependent variables (= arrestment times) to make sure that nei- ther the order of the parasitoids nor the individual sample had a significant influence on their response. However, in none of the experiments a significant effect of order or sample was found indicating that multi-used odor sources elicited constant responses in the parasitoids during the experimental period. The arrestment time on the weevilinfested grains and the number of drumming series and drillings in experiment 2 were analyzed by the KruskalWallis $H$-test followed by multiple Bonferroni-corrected Mann-Whitney $U$-tests for individual comparisons (data partially not normally distributed). Number of offspring (experiment 3) was compared by a one-way ANOVA and subsequent least significant difference (LSD) tests for post hoc comparison. Tibia lengths were analyzed by a twoway ANOVA (factor 1: treatment, factor 2: sex). Statistical analyses except for the GEE-GLM were done using Statistica 4.5 scientific software (StatSoft, Hamburg, Germany).

\section{Competing interests}

The author(s) declare that they have no competing interests.

\section{Authors' contributions}

SS participated in the design of the experiments, performed the chemical analyses and some of the behavioral experiments, and drafted the manuscript. DE performed some of the behavioral and fecundity experiments and did the microbiological part of the study. JLM contributed substantially to the design of the experiments. JR designed the experiments and helped to draft the manuscript. All Authors read and approved the final manuscript.

\section{Acknowledgements}

We are grateful to Verena Ristau and Helgard Nirenberg from the Biologische Bundesanstalt für Land- und Forstwirtschaft, Berlin, Germany for determining the Aspergillus species and for supplying the isolates used in this study. Thomas Hoffmeister (University of Bremen) made useful comments on an earlier draft of the manuscript and kindly performed the GEE-GLM analysis. This research was supported by the Deutsche Forschungsgemeinschaft (DFG grant Ru 7I7/5-I).

\section{References}

I. Jaenike J: On optimal oviposition behavior in phytophagous insects. Theor Popul Biol 1978, I4:350-356.

2. Johnson SN, Birch ANE, Gregory PJ, Murray PJ: The 'mother knows best' principle: should soil insects be included in the preference - performance debate? Ecol Entomol 2006, 3 I:395-40I.

3. Vinson BS, Iwantsch GF: Host suitability for insect parasitoids. Annu Rev Entomol 1980, 25:397-4I9.

4. Sequeira R, Mackauer M: Nutritional ecology of an insect host parasitoid association - the pea aphid Aphidius ervi system.
Ecology 1992, 73:183-189.

5. Meyling NV, Pell JK: Detection and avoidance of an entomopathogenic fungus by a generalist insect predator. Ecol Entomol 2006, 31 1:162-171.

6. Vinson SB: Host selection by insect parasitoids. Annu Rev Entomol 1976, 2 I: 109-133.

7. Godfray HCJ: Parasitoids - Behavioral and Evolutionary Ecology Princeton: Princeton University Press; 1976.

8. Quicke DLJ: Parasitic wasps London: Chapman and Hall; 1997.

9. Schmidt JM: The role of physical factors in tritrophic interactions. Redia 1991, 74:43-93. 
10. Vet LE, Dicke M: Ecology of infochemical use by natural enemies in a tritrophic context. Annu Rev Entomol 1992, 37:|41-172.

II. Vinson SB: Habitat location. In Semiochemicals: their role in pest control Edited by: Nordlund DA, Jones RL, Lewis WJ. New York: John Wiley; 1981:51-77.

12. Tumlinson JH, Turlings TCJ, Lewis WJ: The semiochemical complexes that mediate insect parasitoid foraging. Agric Zool Rev 1992, 5:221-252.

13. Steidle JLM, van Loon JJA: Chemoecology of parasitoid and predator oviposition behaviour. In Chemoecology of insect eggs and egg deposition Edited by: Hilker M, Meiners T. Berlin: Blackwell; 2002:29|-317.

14. Fransen J], van Lenteren JC: Host selection and survivalof the parasitoid Encarsia formosa on greenhouse whitefly, Trialeurodes vaporariorum, in the presence of hosts infected with the Aschersonia aleyrodis. Entomol Exp Appl 1993, 69:239-249.

15. Hoffmeister TS, Roitberg BD: Counterespionage in an insect herbivore-parasitoid system. Naturwissenschaften 1997, 84: I I7-II9.

16. Doumbia M, Hemptinne JL, Dixon AFG: Assessment of patch quality by ladybirds: role of larval tracks. Oecologia 1998 I 1 3:197-202.

17. Frechette B, Dixon A, Alauzet C, Hemptinne JL: Age and experience influence patch assessment for oviposition by an insect predator. Ecol Entomol 2004, 29:578-583.

18. Steidle JLM, Schöller M: Fecundity and ability of the parasitoid Lariophagus distinguendus (Hymenoptera: Pteromalidae) to find larvae of the granary weevil Sitophilus granarius (Coleoptera: Curculionidae) in bulk grain. J Stored Prod Res 2002, 38:43-53.

19. Steidle JLM: The biology of $L$. distinguendus: a natural enemy of stored product pests and potential candidate for biocontrol. IPSP IOBC Bull 1998, 21:103-109.

20. Steidle JLM, Schöller M: Olfactory host location and learning in the granary weevil parasitoid Lariophagus distinguendu (Hymenoptera: Pteromalidae). I Insect Behav 1997, 1 0:331-342.

21. Ruther J, Steidle JLM: Mites as matchmakers: semiochemicals from host-associated mites attract both sexes of the parasitoid Lariophagus distinguendus. I Chem Ecol 2000, 26:1205-1217.

22. Steidle JLM: Host recognition cues of the granary weevil parasitoid Lariophagus distinguendus. Entomol Exp Appl 2000, 95:185-192.

23. Steidle JLM, Fischer A: Quantity does matter: how feces are used for host stage selection by granary weevil parasitoid Lariophagus distinguendus. J Chem Ecol 2000, I 2:2657-2664.

24. Steidle JLM, Ruther J: Chemicals used for host recognition by the granary weevil parasitoid Lariophagus distinguendus. J Chem Ecol 2000, 26:2665-2675.

25. Steidle JLM, Steppuhn A, Reinhard J: Volatile cues from different host complexes used for the host location by the generalist parasitoid Lariophagus distinguendus (Hymenoptera: Pteromalidae). Basic Appl Ecol 200I, 2:I-7.

26. Steidle JLM, Lanka J, Müller C, Ruther J: The use of infochemicals in a generalist parasitoid. Oikos 200I, 95:78-86.

27. Steidle JLM, Steppuhn A, Ruther J: Specific foraging cues used by a generalist parasitoid. JChem Ecol 2003, 29: I31-|43.

28. Hase A: Zur Kenntnis wirtschaftlich wichtiger Tierformen. I Über den Stech- und Legeakt, sowie über den Wirtswechse von Lariophagus distinguendus. Chalcididae. Pteromalini. Naturwissenschaften 1924, 20:377-384.

29. Sinha RN, Wallace HAH: Ecology of insect-induced hot spots in stored grain in western Canada. Res Popul Ecol 1966, 8:107-132.

30. Sinha RN: Insects and mites associated with hot spots in farm stored grain. Can Entomol 1961, 93:609-621.

31. Eighme LE: Relationship of insects to hot spots in stored wheat. J Econ Entomol 1966, 59:564-569.

32. Sunesson AL, Vaes WHJ, Nilsson CA, Blomquist G, Anderson B, Carlson $R$ : Identification of volatile metabolites from five funga species cultivated on two media. Appl Environ Microbiol 1995, 61:2911-2918.

33. Kaminski E, Stawicki S, Wasowicz E: Volatile flavor compounds produced by molds of Aspergillus, Penicilium, and Fungus imperfecti. Appl Microbiol 1974, 27:1001-1004.

34. Chitarra GS, Abee T, Rombouts FM, Posthumus MA, Dijksterhuis ]: Germination of Penicilium paneum conidia is regulated by Iocten-3-ol, a volatile self-inhibitor. Appl Environ Microbiol 2004 70:2823-2829.

35. Samson RA: Occurence of moulds in modern living and working environments. Eur J Epidemiol 1985, I:54-6I.

36. Wilkins CK, Scholl S: Volatile metabolites of some barley storage molds. Int J Food Microbiol 1989, 8: I I-I7.

37. Börjesson T, Stöllman U, Schnürer J: Volatile metabolites produced by six fungal species compared with other indicators of fungal growth on cereal grains. Appl Environ Microbiol 1992 , 58:2599-2605.

38. Menetrez MY, Foarde KK: Microbial volatile organic compound emission rates and exposure model. Indoor Built Environ 2002 I I:208-2 I 3 .

39. Jelen $\mathrm{H}$, Wasowicz E: Volatile fungal metabolites and their relation to the spoilage of agricultural commodities. Food Rev Int 1998, 14:39|-426

40. Fischer G, Schwalbe R, Möller M, Ostrowski R, Dott W: Species-specific production of microbial volatile organic compounds (MVOC) by airborne fungi from a compost facility. Chemos phere 1999, 39:795-810.

41. Atalla MM, Hassanein NM, El-Beih AA, Youssef YAG: Mycotoxin production in wheat grains by different Aspergilli in relation to different relative humidities and storage periods. Nahrung 2003, 47:6-10.

42. El-Shanawany AA, Mostafa ME, Barakat A: Fungal populations and mycotoxins in silage in Assiut and Sohag governorates in Egypt, with a special reference to characteristic Aspergilli toxins. Mycopathologia 2005, I 59:281-289.

43. van den Assem J, van lersel JJA, Los-den Hartogh RL: Is being large more important for female than male parasitic wasps? Behaviour 1989, 108:160-195.

44. Perttunen V: Humidity and light reactions of Sitophilus granarius L., S. oryzae L. (Col., Curculionidae), Rhyzoperta dominica F. (Bostrychidae), and Acanthoscelides obtectus Say (Bruchidae). Ann Ent Fenn 1972, 38:161-176.

45. Steidle JLM, Reinhard J: Low humidity as a cue for habitat preference in the parasitoid Lariophagus distinguendus. Biocontrol 2003, 48: 169-I75

46. Thibout E, Guillot JF, Auger J: Microorganisms are involved in the production of volatile kairomones affecting the host seeking behaviour of Diadromus pulchellus, a parasitoid of Acrolepiopsis assectella. Physiol Entomol 1993, 18:176-182.

47. Thibout E, Guillot JF, Ferary S, Limouzin P, Auger J: Origin and identification of bacteria which produce kairomones in the frass of Acrolepiopsis assectella (Lep., Hyponomeitoidea). Experientia 1995, 51:1073-1075.

48. Madden JL. Behavioural response of parasites to the symbiotic fungus associated with Sirex noctilio. Nature 1968, 21 8: 189-191.

49. Raps A, Vidal S: Indirect effects of an unspecialized endophytic fungus on specialized plant-herbivorous insect interactions. Oecologia 1998, I | 4:54|-547.

50. Rostas M, Simon M, Hilker : Ecological cross-effects of induced plant responses towards herbivores and phytopathogenic fungi. Basic Appl Ecol 2003, 1:43-62.

5I. Cardoza YJ, Lait CG, Schmelz EA, Huang J, Tumlinson JH: Fungusinduced biochemical changes in peanut plants and their effect on development of beet armyworm, Spodoptera exigua Hubner (Lepidoptera: Noctuidae) larvae. Environ Entomol 2003. 32:220-228.

52. Cardoza YJ, Teal PEA, Tumlinson JH: Effect of peanut plant fungal infection on oviposition preference by Spodoptera exigua and on host-searching behavior by Cotesia marginiventris. Environ Entomol 2003, 32:970-976.

53. Rostas M, Ton J, Mauch-Mani B, Turlings TCJ: Fungal infection reduces herbivore-induced plant volatiles of maize but does not affect naive parasitoids. I Chem Ecol 2006, 32:1897-1909.

54. Winston PW, Bates DH: Saturated solutions for the control of humidity in biological research. Ecology 1960, 41:232-237.

55. Ruther J, Homann M, Steidle JLM: Female derived sex pheromone mediates courtship in the parasitoid Lariophagus distinguendus. Entomol Exp Appl 2000, 96:265-274.

56. Kazmer DJ, Luck RF: Field tests of the size-fitness hypothesis in the egg parasitoid Trichogramma pretiosum. Ecology 1995, 76:4I2-425.

57. Ellers J, van Alphen JJM, Sevenster JG: A field study of size-fitness relationships in the parasitoid Asobara tabida. I Animal Ecol 1998, 67:318-324. 